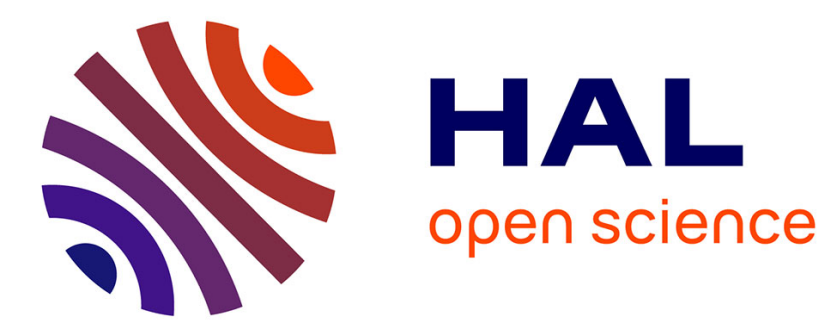

\title{
Classification, based on energetics, of ideal icosahedral clusters, and building blocks of icosahedral quasicrystals and approximant crystals \\ N Tamura, J.-L. Verger-Gaugry
}

\section{- To cite this version:}

N Tamura, J.-L. Verger-Gaugry. Classification, based on energetics, of ideal icosahedral clusters, and building blocks of icosahedral quasicrystals and approximant crystals. Journal of Non-Crystalline Solids, 1993, 153\&154, pp.546-551. 10.1016/0022-3093(93)90412-Q . hal-03134327

\author{
HAL Id: hal-03134327 \\ https://hal.science/hal-03134327
}

Submitted on 10 Feb 2021

HAL is a multi-disciplinary open access archive for the deposit and dissemination of scientific research documents, whether they are published or not. The documents may come from teaching and research institutions in France or abroad, or from public or private research centers.
L'archive ouverte pluridisciplinaire HAL, est destinée au dépôt et à la diffusion de documents scientifiques de niveau recherche, publiés ou non, émanant des établissements d'enseignement et de recherche français ou étrangers, des laboratoires publics ou privés. 


\title{
Classification, based on energetics, of ideal icosahedral clusters, and building blocks of icosahedral quasicrystals and approximant crystals
}

\author{
N. Tamura and J.-L. Verger-Gaugry \\ LTPCM/ENSEEG/INPG (CNRS UA 29), BP 75, Domaine Universitaire, 38402 Saint \\ Martin d'Hères, France
}

\begin{abstract}
We have classified, by their energy, compact finite clusters of icosahedral symmetry, composed of a single atom type, constituted by at most ten layers, and centered at an empty or occupied site with coordination number 12 . The clusters are formed by adding one by one the best possible icosahedral layers in the surface potential minima and by relaxing numerically the cluster at each step. Polyatomic clusters are also considered. The energy is calculated from two-body pair potentials depending only upon the distance. Comparison is made between (1) the succession of layers inside these ideal icosahedral clusters, (2) the layers constituting the real clusters present in m35-approximant crystals and (3) the icosahedral local order in icosahedral quasicrystals as deduced from the 'experimental' atomic surfaces. After revisiting many $\mathrm{m} \overline{35}$-approximant crystals, we propose a characterization and a classification of local icosahedral order in crystallized matter and icosahedral quasicrystals.
\end{abstract}




\section{Introduction}

It is now known that the icosahedral local order in icosahedral quasicrystals and some $m \overline{3} \overline{5}$ approximant crystals in a given system is fundamentally the same, up to very small deformations $[1,2]$. In the $\mathrm{i}$-phase, the diffraction process is dominated (1) by the diffraction properties of the different pseudoicosahedral clusters of atoms formed locally (pseudoinflation rules, pseudoicosahedral invariance, etc.), and (2) by the quasiperiodic way these pseudoicosahedral clusters assemble and interpenetrate [3] to provide long range orientational order, and the diffraction properties of quasiperiodicity [4]. The quasiperiodic distribution of the clusters is dictated by the structure of the possible icosahedral clusters which can be formed in a definite system, where cluster centers cannot be at any atomic site [5]. In ref. [6], Henley has classified the various icosahedral phases by classes, but his classifica- tion is essentially done by systems, and in fact, by the types of icosahedral local order the systems 'can offer'. It is therefore relevant to determine the different possible types of icosahedral local order which can occur in a system, that is, the different possible icosahedral atomic configurations of clusters of lowest energy which can be formed and are stable with a small number of atom types, by (1) starting to obtain ideal clusters, and (2) comparing with pseudoicosahedral clusters in binary i-phases and approximant crystals.

After recalling Henley's classification [6], we investigate ideal icosahedral atomic clusters, then propose a realistic classification of real pseudoicosahedral clusters as they occur in approximant crystals, and finally discuss the possible building blocks for both approximant crystals and i-phases.

\section{Icosahedral order in icosahedral quasicrys- tals: Henley's classes}

For icosahedral quasicrystals, the situation seems a priori simpler than in approximant crys- 
tals, since there exists only one way to define the action of the icosahedral group in reciprocal space, which amounts to having only one orientational family of pseudoicosahedral clusters in real space [7,8]; the presence of quasiperiodicity in the i-phase eliminates the possibility of several coexisting orientational families of pseudoicosahedral clusters, families which, on the contrary, generally coexist in the case of approximant crystals (see below and, for instance, ref. [8] for the Ti-TM system). In ref. [6], Henley has reported i-phases and approximant crystals, in various sys- tems, and cluster packing geometries with smallsized (i.e. limited to a few layers) clusters as follows:

- the Al-TM class, where TM is a transition metal and $\mathrm{Al}$ in substitution by other elements: Pm $\overline{3} \overline{5}$ type: i-AlMn, i-AlMnSi, i-AlGeMn, i$\mathrm{AlCr}, \mathrm{i}-\mathrm{AlCrSi}$, i-AlV, ... F Fm $\overline{3} \overline{5}$ type: i-Al$\mathrm{FeCu}$, i-AlPdMn, i-AlCuOs, i-AlCuRu, ...;

- the AlMgZn class: i-AlLiCu, i-AlMgZn, i-Al$\mathrm{LiZn}, \mathrm{i}-\mathrm{AlAgMg}, \ldots$ (i-GaMgZn);

- the Ti-TM class: i-TiMn, i-TiCo, i-TiFe,...;

- the i-PdUSi class.

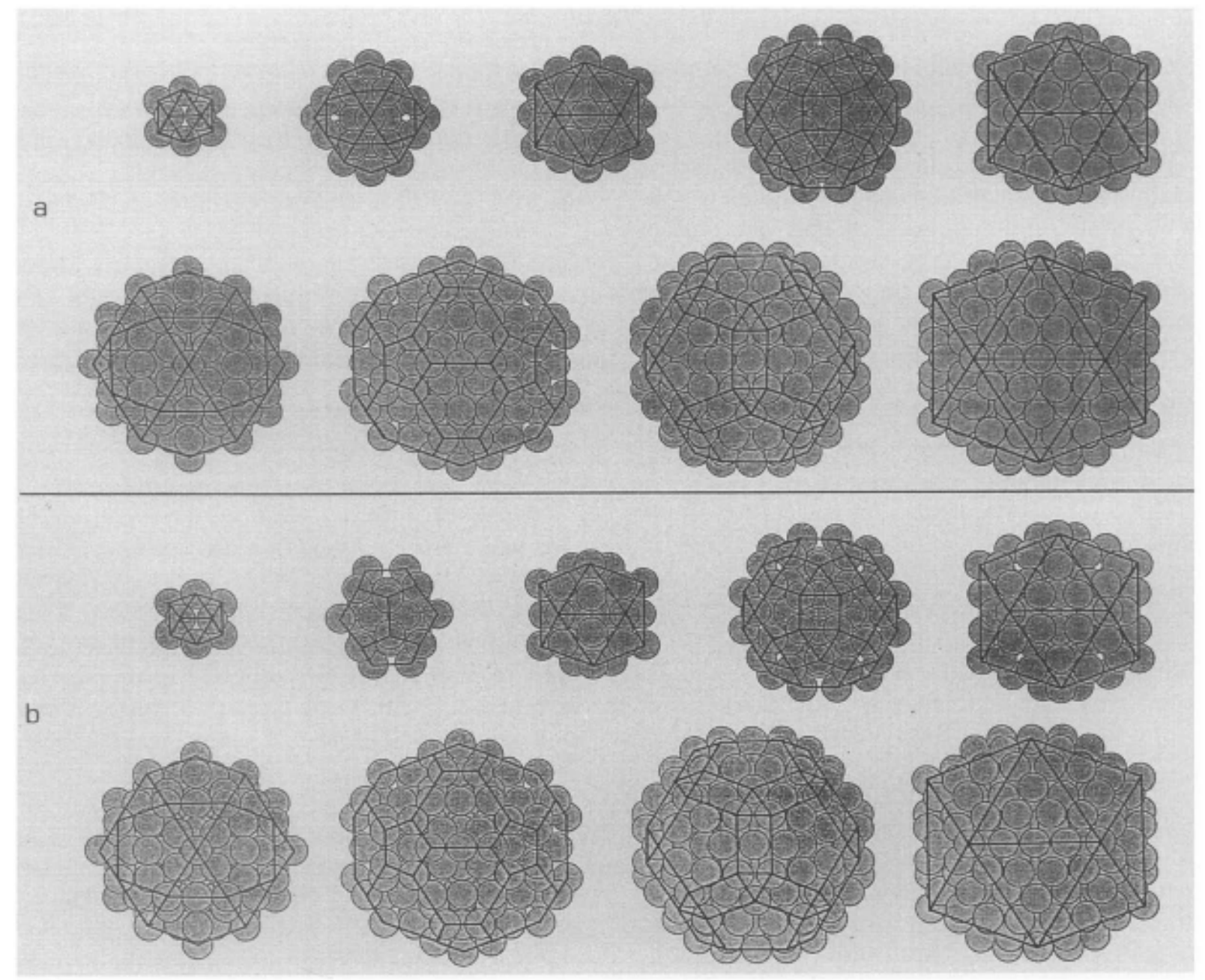

Fig. 1. Succession of icosahedral layers in two energetically favourable monatomic clusters: (a) sequence 1, starting by a Mackay icosahedron, (b) sequence 4 starting by a Bergmann unit. The center is occupied or not by an atom (the other sequences are not represented for lack of space). 
These phases may coexist with decagonal phases, having common symmetry axes. The pseudoicosahedral clusters, having a chemical disorder or not [9], when slightly flattened along the tenfold axis, now become pseudocyclic or pseudodihedral units in the d-phase.

The primitive character of $\mathrm{i}-\mathrm{AlMnSi}, \mathrm{i}-\mathrm{AlCr}, \ldots$ phases corresponds to one icosahedral environment type. On the contrary, fcc icosahedral structures like $\mathrm{i}-\mathrm{AlFeCu}, \mathrm{i}-\mathrm{AlCuRu}$ are compatible with the existence of at least two local icosahedral environments, the second one being either a chemical variation of the first one (i-AlPdMn, [10]) or different (i-AlFeCu, [11]).

\section{Classification of ideal icosahedral building blocks}

We are interested in stable clusters having exactly the icosahedral symmetry $m \overline{3} \overline{5}$ as a symmetry group, and composed of only one type of atom. Stable means that, whatever the (small) displacements we impose on the atomic sites, the cluster recovers entirely the icosahedral symmetry by numerical relaxation [18], with the same geometry as in the initial state (relative energy minimum). The energy is calculated by means of a normalized 6-12 Lennard-Jones pair potential depending only upon the distance.

The construction process for the clusters is the following:

\subsection{Monoatomic clusters}

Starting from a central site which could be unoccupied, we successively add icosahedral atomic-site polyhedra (one atom at each vertex). The surface potential and the configuration energy of the cluster are calculated at each step. The atoms of the added shell are positioned at the minima of the surface potential, respecting each time the icosahedral symmetry. The cluster is then relaxed, and so on up to tenth neighbors. We always start from $\mathrm{CN} 12$ sites (icosahedron as first layer). For the second and third neighbors, we have only two possibilities: the sequence ico1-icosi1-ico2 (Mackay icosahedron) and the sequence ico1-dodeca1-ico2 (Bergmann unit). For the fourth neighbors and above, the number of possibilities increases rapidly. But, at the final step (tenth neighbors), many cases can be eliminated because the configuration energy is too high or there is a too low density around some atoms in the inner shells. This elimination reduces the number of possible icosahedral clusters. The following sequences of icosahedral layers represent the succession of layers inside the more energetically favourable clusters, assumed to be surrounded by vacuum:

1) ico1-icosi1-ico2-hexe1-ico3-icosi2-pentakis1-hexe2-ico4 (fig. 1(a));

2) ico1-icosi1-ico2-dodeca1-pentakis-1-ico3trisico1-hexe1-pentakis2;

3) ico1dodeca1-ico2-pentakis1-dodeca2-ico3hexe1-icosi1-pentakis2;

4) ico1-dodeca1-ico2-hexe1-ico3-icosi1-pentakis1-hexe2-ico4 (fig. 1(b));

5) ico1-dodeca1-ico2-icosi1-hexe1-ico3-trisico1-pentakis1-hexe2.

The other cluster energies are singularly higher, and only these five sequences are relevant. The succession of radii is not given since a slight change in the eV-calibration of the potentials modifies them.

\subsection{Polyatomic clusters}

Atoms of different sizes are now considered. We proceed by reference to the above classification; we introduce no chemical disorder in the cluster with two or three kinds of atoms. These new atoms have a reasonable difference of size with respect to the first one (ratio of the radii between 0.75 and 1). A layer in a cluster is then monoatomic and of perfect icosahedral symmetry. With two types of atoms, some configurations are more stable than others: sequence 1 is favoured, and it is the situation encountered in the $\alpha$-Al$\mathrm{MnSi}$ type phases with $\mathrm{Al}$ and transition metals; sequence 3 enters in competition with sequence 1 , is energetically less favored, and probably does not occur. Sequence 3 corresponds to the R-phase type local order but with constitutive atoms having a large difference of size; sequences 2 and 5 are also energetically favored and may exist in 
hypothetical icosahedral or approximant crystals. $\mathrm{Ni}$ environments in $\eta-\mathrm{Ti}_{2} \mathrm{Ni}$ and some $\mathrm{Mn}$ environments in $\mu-\mathrm{Al}_{4} \mathrm{Mn}$ are given by sequence 5 which is close to the $\alpha$-phase type $[8,12]$. Therefore, among the five above sequences, only four of them remain physically reasonable. In this approach, the discriminator for the classification is the energy, but the energy calculations, with real atoms, are extremely complicated and should be done by ab initio methods [13]. The present construction process with Lennard-Jones potentials is just a guide which provides possible stable atomic configurations with no chemical disorder with respect to the icosahedral symmetry.

\section{Real icosahedral local order in $\mathbf{m} \overline{3} \overline{5}$-approxi- mant crystals}

The approximant crystals prototypes are: 1) the $\alpha$-phase type: ico1(Al)-icosi1(Al)-ico2(TM)hexe1( $\mathrm{Al})$-ico3( $\mathrm{Al})$-icosi2( $\mathrm{Al}, \mathrm{TM})$-pentakis1 (Al, TM)-incomplete hexe2( $\mathrm{Al})$, and incomplete trisico1(Al)-ico4(Al). Other larger polyhedra can be defined: an arithmetic sequence of 17 icosahedra, a geometric sequence of 10 icosidodecahedra (inflated by the golden mean), etc. [3,12]. The body centered site is surrounded by a similar environment but hexe1 is somewhat distorted whereas ico3 is replaced by an incomplete dodecahedron. The $\alpha$-phase structure is a $\mathrm{CsCl}$ type stacking of Mackay icosahedra (54 atoms) at body centered sites, and a larger cluster defined by the above sequence truncated at ico3 (126 atoms), at the origin sites; 2) the R-phase type: ico1(Al)dodeca1(Li)-ico2(Al)-pentakis1(Al)-dodeca2(Li) -ico3(Li)-incomplete icosi1( $\mathrm{Al})$-hexe1( $\mathrm{Al}, \mathrm{Li})$ $\ldots$ at body centered sites and origin sites. The structure is described by a bec stacking of the cluster obtained by truncating the above sequence at the ico3. To emphasize size effects, we have, for the building block: ico1(MA)dodeca1(LA)-ico2(MA)-pentakis1(LA, MA)dodeca2(LA)-ico3(LA), where LA means large sized atom $(\mathrm{Li}, \mathrm{Mg}, \ldots)$ and $\mathrm{MA}$ middle sized atom $(\mathrm{Al}, \mathrm{Zn}, \ldots)$. It is a fact that a strong interpenetration of the big clusters actually occurs in both phases.
From investigations on many approximant crystals having local atomic configurations exhibiting almost the icosahedral symmetry and for which this symmetry spreads far inside the crystal from certain centers (which are occupied or empty sites), we propose the following classification scheme; the approximant crystals are characterized by:

- the number of pseudoicosahedral clusters (counted by their center) per unit cell of the Bravais lattice;

- the cluster type, which is, starting from their centers, chosen from the above sequences 1 to 5 (section 3), and is either of Mackay type: ico1-icosi1-ico2-..., or of Bergmann type: ico1-dodeca1-ico2-...;

- the number of icosahedral orientations in the whole family of clusters; one orientational family is constituted by only one type of cluster of the same orientation. (It is the number of non $\mathrm{m} \overline{3} \overline{5}$-equivalent ways the action of the icosahedral group can be coherently defined in the basis of the Bravais lattice [7]);

- the degree of interpenetration of the clusters [3];

where the cluster type is specified by:

- the occupation or not of the center of the cluster by an atom;

- the succession of the average radii of the (pseudoicosahedral) layers;

- the spatial extent of the cluster in the approximant crystal;

- the deformation, global and by layer, with respect to a perfect icosahedral cluster optimally positioned in the orientational family of the cluster [7,14];

- the degree of completeness of the pseudoicosahedral layers, and the layer from which empty sites begin to appear;

- the types of atoms in the layers;

- the chemical disorder of the cluster with respect to the icosahedral symmetry, which is measured, on a complete layer, by the proportion of different atoms (where we assume that the action of the icosahedral group is transitive), while a cluster without any chemical disorder corresponds to the situation where each layer exhibits only one type of atoms; 
- the existence, inside and outside the cluster, of arithmetic integral sequences (the $n$th layer is $n$ times farther than the first one) and of geometric sequences (the $(n+1)$ th one is $t^{n}$ times farther than the first one, where $t$ is a real number $>1$ ) of homothetic concentric layers (homothety is exact, 'up to small deformations').

In fact, a systematic feature of icosahedral local order in approximant crystals associated to $\mathrm{i}$-phases is that the first layers are positioned as in Mackay type clusters (ico1-icosi1-ico2, $\alpha$-Al$\mathrm{MnSi}$ phase type) or in Bergmann type clusters (ico1-dodeca1-ico2, R-AlLiCu phase type), with or without an atom at the center. No other possibility was observed and this result is coherent with the classification of ideal clusters in section 3 . The local environment of the $\alpha$-phase type until ico3 is perfectly recognizable around $\mathrm{Cr}$ sites ( $=$ cluster centers) in $\theta-\mathrm{Al}_{45} \mathrm{Cr}_{7}$ associated with $\mathrm{i}-\mathrm{AlCr}$, around $\mathrm{Mn}$ sites in hexagonal $\beta$ $\mathrm{Al}_{9} \mathrm{Mn}_{3} \mathrm{Si}$ associated with $\mathrm{i}-\mathrm{AlMnSi}$, around some Mn sites in hexagonal $\mu-\mathrm{Al}_{4} \mathrm{Mn}$ associated with $\mathrm{i}$-AIMn, around some $\mathrm{V}$ atoms in cubic $\alpha-\mathrm{Al}_{10} \mathrm{~V}$, monoclinic $\alpha^{\prime}-\mathrm{Al}_{45} \mathrm{~V}_{7}$ and hexagonal $\beta-\mathrm{Al}_{23} \mathrm{~V}_{4}$, associated with $\mathrm{i}-\mathrm{AlV}$, around $\mathrm{Fe}$ atoms in hexagonal $\alpha^{\prime}$-AlFeSi and cubic $\alpha$-AlFeSi. Some phases which have no icosahedral short range order nevertheless have regions of the $\alpha$-phase type environment: the monoclinic $\theta-\mathrm{Al}_{13} \mathrm{Fe}_{4}$ phase associated with $\mathrm{i}-\mathrm{AlFeCu}$ and $\mathrm{d}-\mathrm{AlFe}$, thus appearing as a link between i- and d-phases [9], the orthorhombic o- $\mathrm{Al}_{4} \mathrm{Mn}$ and $\alpha-\mathrm{Al}_{23} \mathrm{CuFe}_{4}$, respectively, associated with $\mathrm{i}-\mathrm{AlMn}$ and $\mathrm{i}-\mathrm{AlFeCu}$.

As for the R-phase type local environment, it exists in the isostructural $\mathrm{T}-(\mathrm{Al}, \mathrm{Zn})_{49} \mathrm{Mg}_{32}$, $\mathrm{Al}_{6} \mathrm{CuMg}_{4}$ and $\mathrm{Al}_{6} \mathrm{ZnLi}_{3}$ phases, respectively, associated with $\mathrm{i}-\mathrm{AlZnMg}, \mathrm{i}-\mathrm{AlCuMg}$ and $\mathrm{i}-\mathrm{Al}$ $\mathrm{ZnLi}$.

In $\mathrm{c}-\mathrm{Ti}_{2} \mathrm{TM}$, around $\mathrm{Ti}(\mathrm{c})$ and $\mathrm{TM}(\mathrm{e})$ atoms, we have a (Bergmann) sequence, resp. truncated at the 6th and 4th neighbors, of type 5, with a strong chemical disorder already on the first layer, respectively: $3 \mathrm{Ni}$ for $9 \mathrm{Ti}, 6 \mathrm{Ni}$ for $6 \mathrm{Ti}$, and therefore large deformations. Still more deformed, the UPdSi phase enters into the case of phases where icosahedral local order is formed from atoms of very different sizes, and too few results are now known on such occurrences to compare usefully to the i-UPdSi class local order.

\section{Comments}

When no chemical disorder happens in clusters, all the layers correspond to crystallographic orbits in an $n \mathrm{D}$ Euclidean hyperspace $(n=6$ currently), and this correspondence outlines the importance of models of i-phases given by sphere fibrations [15] and an $n \mathrm{D}$ space group. When holes appear in clusters in some layers, this means that a local zero curvature at these layers (in 3D space) is not suitable for obtaining the i-phase from the $n \mathrm{D}$ crystal, and we have geometric frustration [16]. The present approach emphasizes the spatial extent of clusters, more than the notions of glue atoms and polyhedra (octahedra, etc.) for linking clusters which become meaningless.

The three approaches converge towards the same classification. We have obtained a limited number (4) of stable icosahedral clusters with slightly different atomic sizes. Two of them correspond to the i-phases related to the $\alpha$-phase and to the R-phase. This correspondence implies that the number of icosahedral phase types is necessarily limited by the small number of possible icosahedral local orders in metallic compounds. It is not reasonable to think that several icosahedral phases of different space group types coexist with the same approximant crystal in a given system, as it was suggested in ref. [17], since given atom types seem to force unique icosahedral local atomic configurations and linkages between the clusters, therefore, a unique i-phase, and unique diffraction properties in quasiperiodic conditions. However, chemical and topological disorders inside clusters and in packing rules, as far as interpenetration of clusters allows to describe them in a simple way, occur, as they occur in approximant crystals.

\section{Conclusion}

We have derived possible ideal icosahedral clusters and have compared them with the icosa- 
hedral local order, as it appears in approximant crystals. Comparison is also made with a classification of icosahedral phases, based on the icosahedral local order.

\section{Abbreviations}

ico $=$ icosahedron; dodeca $=$ dodecahedron; icosi = icosidodecahedron; trisico $=$ trisicosahedron; hexe $=$ hexecontahedron; pentakis $=$ pentakisdodecahedron (soccer ball); TM = transition metal.

\section{References}

[1] D.P. Divicenzo and P.J. Steinhardt, Directions in Condensed Matter Physics, Vol. 11: Quasicrystals, The State of the Art (World Scientific, Singapore, 1991).

[2] Aperiodicity and Order, Extended Icosahedral Structures, Vols. 1-3, eds. M.V. Jaric and D. Gratias (Academic Press, New York, 1989).

[3] J.-L. Verger-Gaugry, Int. Symp, on the Physics and Chemistry of Finite Systems: From Clusters to Crystals, Richmond, Virginia, 1991 (Kluwer Academic, 1992) Nato Series; in: Methods of Structural Analysis of Modulated Structures and Quasicrystals, eds. J.M. Perez-Mato, F.J. Zuniga and G. Madariaga (World Scientific, Singapore, 1991) p. 637.

[4] A. Katz and M. Duneau, J. de Phys. 47 (1986) 181.

[5] J.L. Aragon, D. Romeu and A. Gomez, in: Methods of Structural Analysis of Modulated Structures and Qua- sicrystals, eds. J.M. Perez-Mato, F.J. Zuniga and G. Madariaga (World Scientific, Singapore, 1991) p. 648.

[6] C.L. Henley in: Quasicrystals, eds. T. Fujiwara and T. Ogawa, Springer Series in: Solid-State Sciences 93 (Springer, Berlin, 1990) p. 38

[7] J.-L. Verger-Gaugry, I, J. Phys. I France, 1 (1991) 1303.

[8] N. Tamura, A. François, A. Loiseau and J.-L. VergerGaugry, submitted to Philos. Mag. B.

[9] J.-N. Barbier, N. Tamura and J.-L. Verger-Gaugry, J. Non-Cryst. Solids 153\&154 (1993) 126.

[10] M. Boudard, C. Janot, M. de Boissieu, J.-M. Dubois and C. Dong, in: Methods of Structural Analysis of Modulated Structures and Quasicrystals, eds. J.M. Perez-Mato, F.J. Zuniga and G. Madariaga (World Scientific, Singapore 1991) p. 515.

[11] M. Cornier-Quiquandon, A. Quivy, S. Lefebvre, E. Elkaim, G. Heger, A. Katz and D. Gratias, Phys. Rev, B44 (1991) 2071.

[12] N. Tamura and J.-L. Verger-Gaugry, submitted to Philos. Mag. A.

[13] Int. Symp. on the Physics and Chemistry of Finite Systems: From Clusters to Crystals, Richmond, Virginia, 1991 (Kluwer Academic, 1992) Nato Series.

[14] J.-L. Verger-Gaugry, N. Tamura and J-.N. Barbier, II, J. Phys. I France, to be submitted.

[15] J.-F. Sadoc and R. Mosseri, J. Non-Cryst. Solids 153\&154 (1993) 247

[16] J.-F. Sadoc and R. Mosseri, in: Aperiodicity and Order, Extended Icosahedral Structures, Vol. 3, eds. M.V. Jaric and D. Gratias (Academic Press, New York, 1989) p. 163.

[17] J.-L. Verger-Gaugry, in: Number Theory and Physics, Springer Proceedings in Physics 47, eds. J.-M. Luck, P. Moussa and M. Waldschmidt (Springer, Berlin, 1989) p. 128.

[18] M.R. Hoare, J. Non-Cryst. Solids 31 (1978) 157. 